\title{
Co-Occurrence of Leber Congenital Amaurosis and Meckel Syndrome Type 1 in a Fetus: Is There a Lesson to Be Learned?
}

\author{
Karthik Tallapaka, ${ }^{a, b}$ Shagun Aggarwal ${ }^{a, b} \quad$ Amrita Bhattacherjee $^{b}$ \\ Aneek Das Bhowmik $^{b}$ Ashwin Dalal ${ }^{a}$, \\ aDepartment of Medical Genetics, Nizam's Institute of Medical Sciences, and b Diagnostics Division, Centre for \\ DNA Fingerprinting and Diagnostics (CDFD), Hyderabad, India
}

\section{Established Facts}

- The identification of pathogenic variants not associated with the primary phenotype of a patient is being increasingly reported after the advent of NGS in clinical practice.

- Reporting mutations as secondary findings in NGS analysis is not yet uniformly recommended by professionals.

\section{Novel Insights}

- We describe a rare case in which pathogenic variants causing 2 ciliopathies, Leber congenital amaurosis (LCA) and Meckel syndrome, were identified in a single fetus whose elder sibling was previously diagnosed with LCA by NGS analysis and was also found to carry a pathogenic mutation in the MKS1 gene in heterozygous state by retrospective study.

- It may be of importance to report pathogenic/likely pathogenic variants in heterozygous state identified as a secondary finding in a child with consanguineous parents or when he/she is from a highly inbred community.

\section{Keywords}

Exome sequencing · Leber congenital amaurosis · Meckel syndrome $\cdot$ Secondary findings

\section{Abstract \\ A patient referred for prenatal diagnostics, after first-trimes- ter ultrasound due to a previous child with Leber congenital amaurosis, was suggestive of a Meckel syndrome-like phe-}

notype. Fetal autopsy confirmed the multiple anomalies, and whole-exome sequencing of the fetal DNA identified a pathogenic variant in the RPGRIP1 gene, previously identified in the elder sibling, and a variant causative of Meckel syndrome 1 in the MKS1 gene. Reporting the MKS1 mutation, which was present in heterozygous state in the elder sibling, as a secondary finding would have enabled the parents to be tested for carrier status of the same variant and appropriate counseling could have been provided prior to the onset of

\section{KARGER}

(c) 2019 S. Karger AG, Basel

E-Mail karger@karger.com

www.karger.com/msy
Dr. Shagun Aggarwal

Department of Medical Genetics

Nizam's Institute of Medical Sciences, Panjagutta

Hyderabad 500082 (India)

E-Mail shagun.genetics@gmail.com 
the pregnancy. Although the information may not be of great benefit in cases where the ultrasonographic changes can be recognized early, it would be of definitive help where diagnostic imaging in early pregnancy is not possible.

(c) 2019 S. Karger AG, Basel

With the increasing use of NGS technologies in clinical practice, the identification of pathogenic alleles responsible for 2 or more diseases, especially in patients with complex/compounding phenotypes, is not rare. Herein, we describe a case in which pathogenic variants causing 2 ciliopathies with nonoverlapping phenotypes were identified in a fetus and discuss its implications in reporting secondary findings in NGS-based exome analysis.

\section{Clinical Report and Methods}

A 21-year-old woman (G3P2L1A1) came to our Institute for chorionic villous sampling and targeted mutation analysis in view of a previous child being affected with Leber congenital amaurosis (LCA). A 21-bp homozygous deletion in the RPGRIP1 gene was detected in the child by clinical exome sequencing and was later confirmed by Sanger sequencing. The second pregnancy of the couple ended as a spontaneous abortion after about 3 months of gestation. There was a history of gastroschisis in a cousin and polydactyly with early infantile death in another. This was the third pregnancy of the second-degree consanguineous couple. Fetal sonogram prior to the chorionic villous sampling revealed multiple malformations such as occipital encephalocele, postaxial polydactyly, multicystic dysplastic kidneys, cleft lip, absent nasal bone, mesomelia, and absent tibia and fibula suggesting a Meckel syndrome-like phenotype. The parents were counseled regarding the poor prognosis, and after obtaining the fetal samples, the pregnancy was terminated.

Upon autopsy examination, externally, there was a midline depression in the cranium which was extending onto the forehead. A circular defect was identified in the occipital region of the cranium. There was significant hypertelorism. The nose was not well formed and both lateral maxillary processes were not fused. The nasal process was also not well developed, resulting in a large median upper lip cleft. Cleft palate and micrognathia were evident. The abdomen appeared distended, external genitalia were normal female, and anal opening could not be visualized. All limbs showed postaxial polydactyly and both lower limbs were internally angulated in addition to bilateral congenital talipes equinovarus (Fig. 1A-C). The fetal anthropometric measurements corresponded to a gestational age of 12 weeks. The placenta measured $7 \times 5 \mathrm{~cm}$ and appeared hydropic; a 3-vessel cord was seen.

The intra-abdominal examination revealed enlarged and polycystic kidneys (Fig. 1D). The liver was friable and the sigmoid colon appeared thin. The remaining intra-abdominal organs showed no abnormalities. Normal female internal genitalia were seen. The intrathoracic examination was grossly normal. The heart was without noticeable defects and great vessels were correctly aligned. The
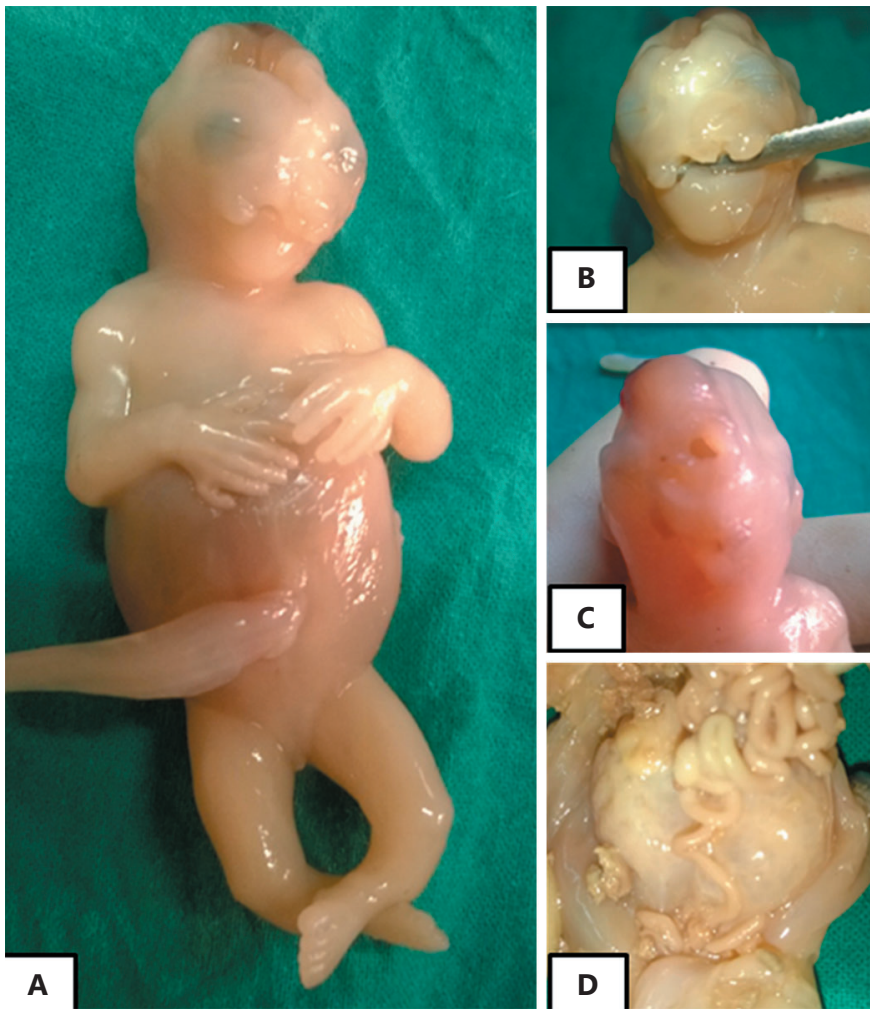

Fig. 1. Fetal autopsy findings. A Photograph of the fetus showing midline groove in the cranium, hypertelorism, cleft lip, postaxial polydactyly, distended abdomen, and bilateral congenital talipes equinovarus. B Median cleft lip. C Occipital defect. D Bilateral enlarged polycystic kidneys.

lungs showed no changes in size, shape, or lobation. The diaphragm was intact, and the trachea and esophagus showed no clinical manifestation. Supratentorial structures could not be detected on intracranial examination; they might have eviscerated during the process of expulsion. The cerebellum was visualized in the posterior fossa. No brain/meningeal herniation was apparent through the occipital defect. Fetal radiographs confirmed the defect in the cranium, bilateral congenital talipes equinovarus, and revealed the presence of hypoplastic tibiae and fibulae.

As the autopsy findings of the fetus were suggestive of a primary ciliopathy, with a Meckel syndrome-like phenotype and because the elder sibling had a homozygous NM_020366.3:c.900_906 \pm 14delTCAAGAGGTGAGTTGCCATCA 21-bp deletion of the RPGRIP1 gene, and mutations in RPGRIP1 are known to cause only ophthalmic involvement, a possibility of oligogenic inheritance in this case with a coexisting pathogenic variant/s in an another ciliopathy gene was considered. The karyotype of the fetus was normal and subsequently, whole-exome analysis was performed by NGS in the stored fetal DNA to elucidate the exact genetic etiology.

Genomic DNA was extracted from the chorionic villus sample of the mother. Exome capture was performed on $3 \mu \mathrm{g}$ of genomic DNA using the Nextera Rapid Capture kit. The library was se- 


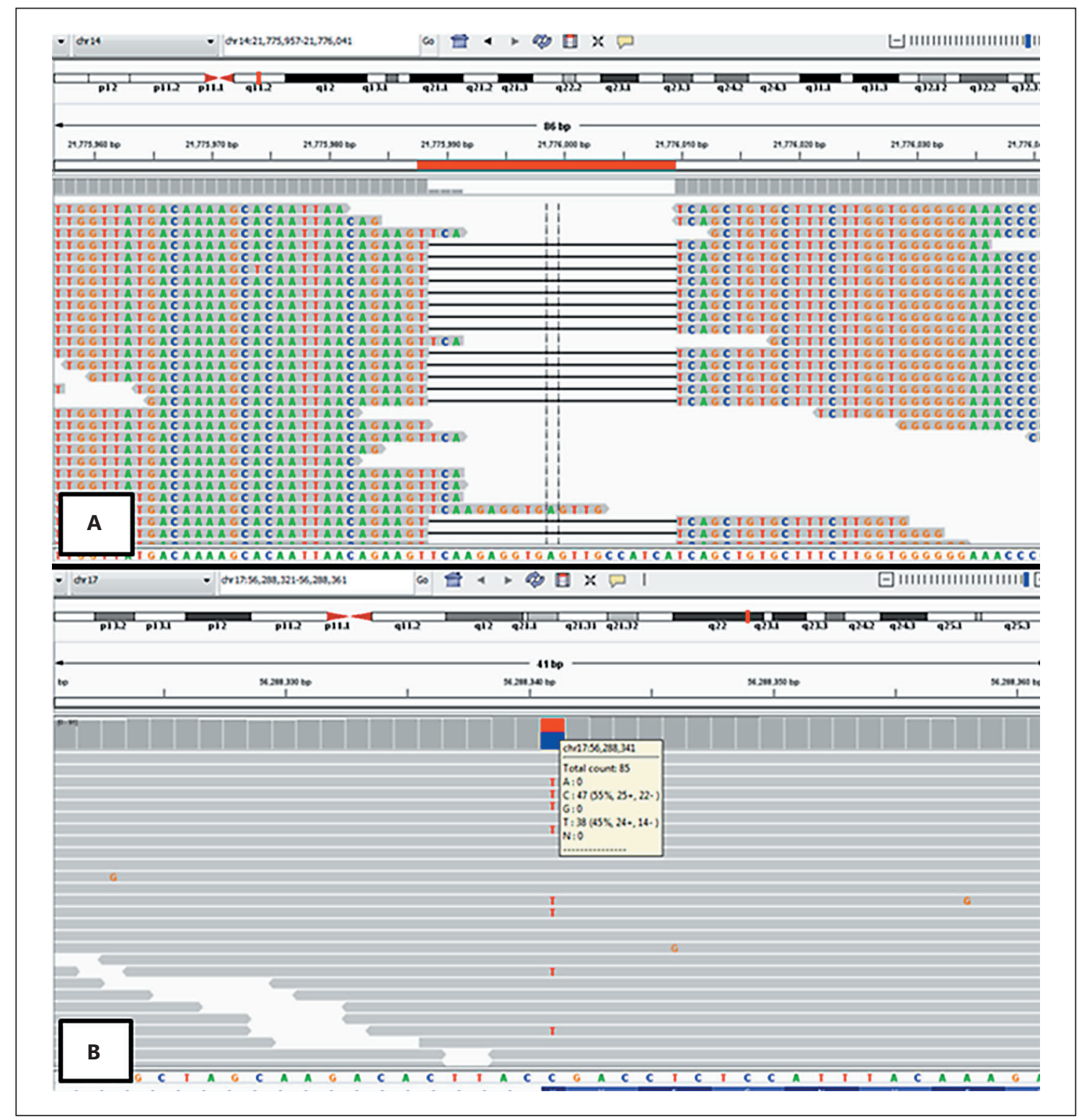

Fig. 2. NGS analysis. A IGV snapshot showing the homozygous 21-bp deletion in RPGRIP1. B IGV snapshot showing the NM_017777.3:c.958G>A heterozygous variant in the MKS1 gene of the elder sibling.

quenced to mean $110 \times$ coverage on the Illumina HiSeq2000 sequencing platform. The sequences obtained were aligned to the human reference genome (GRCh37/hg19) using the BWA program [Li and Durbin, 2010] and analyzed using the Picard and GATK-Lite toolkit [Li et al., 2009; McKenna et al., 2010] to identify variants in the whole exome relevant to clinical indication as described earlier by Das Bhowmik and Dalal [2015]. Variant annotation was completed using ANNOVAR [Wang et al., 2010] for position and predicted function. Filtering was done using 1000 Genomes, exome variant server (EVS), Exome Aggregation Consortium (ExAC) [using a minor allele frequency (MAF) of $\leq 0.01$ ], and dbSNP databases. Clinically relevant mutations were anno-

Two Ciliopathies in a Fetus: Reporting Secondary Findings tated using published variants in the literature and a set of variant databases including ClinVar, OMIM, and the Human Gene Mutation Database (HGMD).

\section{Results}

Exome sequencing revealed a homozygous NM_017777.3:c.958G >A (p.Val320Ile) pathogenic variant in the MKS1 gene responsible for Meckel syndrome I 
(OMIM 249000) in addition to the homozygous 21-bp deletion in the RPGRIP1 gene (Fig. $2 \mathrm{~A}$ ), suggesting 2 ciliopathies in the same fetus. The RPGRIP1 mutation is a novel splice site disrupting variant not reported previously in either 1000 Genome or ExAC databases and is predicted to be pathogenic by in silico mutation prediction software such as MutationTaster and Human Splicing Finder. It affects the invariant GT donor splice site in addition to the deletion of 7 nucleotides in exon 6 . The c. $958 \mathrm{G}>\mathrm{A}$ variant in the $M K S 1$ gene was previously reported as the likely cause in a fetus with Meckel syndrome though functional validation for the same was not done [Khaddour et al., 2007]. This variant is not present in the 1000 Genome database; however, it is present in ExAc and Genome AD databases at a very low frequency of 0.00002486 and 0.00002032 , respectively. It affects the last nucleotide of exon 10 and is most likely to affect the donor splice site. It has a CADD score of 23.8 and is predicted to be disease causing by MutationTaster, SIFT, and PolyPhen-2 in addition to the Human Splicing Finder. Thus, both variants can be classified as pathogenic variants as per American College of Medical Genetics and Genomics (ACMG) and the Association for Molecular Pathology guidelines [Richards et al., 2015].

\section{Discussion}

In this study, we present a rare case in which pathogenic variants causing 2 ciliopathies with nonoverlapping phenotypes were identified in the same fetus. To the best of our knowledge, no such case has been previously reported. Retrospectively, we reanalyzed the raw data of the elder sibling's exome analysis and found that she was carrying the $M K S 1$ pathogenic variant in heterozygous state (Fig. 2B). This prompted us to hypothesize that determining carrier status of pathogenic/likely pathogenic variants in genes unrelated to the patient's phenotype, especially in those children born to consanguineous parents, may be helpful when counseling the affected families.

Attempting this in the first child, we could narrow down 53 variants after stringent filtering considering exonic nonsynonymous, frameshift, or stop-gain variants with an MAF of less than 0.01 in population databases. CADD scores, InterVar predictions, and the presence a characteristic phenotype were further included in the filtering process. Two of the variants in addition to the MKS1 mutation were found to be of reportable significance, all others being classified as variants of unknown significance. These included a heterozygous
NM_000060:c.968A $>\mathrm{G}$ variant in the BTD gene which was earlier reported in patients with biotinidase deficiency. The other was a NM_000918:c.511C > T stopgain variant in the $P 4 H B$ gene which can be classified as a pathogenic variant as per ACMG guidelines. However, as the child did not have any features of Cole Carpenter syndrome 1, an autosomal dominant disease, it is likely that this mutation is benign, at least in the heterozygous state.

The big debate on reporting secondary or incidental findings was addressed by recommendations from ACMG published in 2013, which was later updated in 2016 [Green et al., 2013; Kalia et al., 2017]. The list is dominated by autosomal dominant or X-linked conditions with high penetrance where there is a general consensus regarding the clinical utility of reporting the variants. The ATP7B gene (mutations in this gene cause Wilson disease) is the only autosomal recessive disorder where pathogenic or expected pathogenic variants need to be reported when present in homozygous/compound heterozygous state [Kalia et al., 2017]. Especially among genetic health care providers, reporting incidental/secondary variants with clinical/personal utility has found growing acceptance and can be of importance when it comes to reproductive decision-making for parents [Tabor et al., 2014; Mackley et al., 2017].

In the present case, the parents could have been checked for the MKS1 and BTD gene variants, if their presence in the child with LCA had been reported. Although this might not have been necessary for the prenatal diagnosis of Meckel syndrome, which is a straightforward ultrasonographic diagnosis, it would play a crucial role in detecting biotinidase deficiency, which is not possible by ultrasound. Similar to this family, reporting of carrier status of pathogenic/likely pathogenic variants in genes unrelated to the patient's phenotype would be of benefit to the communities which are largely inbred and where consanguinity is a common practice, and therefore, the a priori risk of having a partner with the same variant is high. Though this is bound to increase the burden on bioinformatics services and complicate genetic counseling processes, it would be imprudent to ignore the benefits of reporting such variants on reproductive decision making of reporting on reproductive decision-making. The present study shows the necessity to report secondary/incidental findings of pathogenic or likely pathogenic mutations in children living in inbred communities and/or with consanguineous parents and considering the carrier status of parents in genetic counseling and family planning. 


\section{Acknowledgments}

We would like to acknowledge Dr. Vijayalaksmi for the clinical evaluation of the child with LCA. We would also like to express our gratitude to the reviewers, whose suggestions have been invaluable in improving the manuscript.

\section{Statement of Ethics}

This study has been approved by the Institutional Ethics Committee and informed consent was obtained from the parents for publication of this case report and accompanying images as well as molecular analysis.

\section{Funding Sources}

Molecular work was supported by a research grant from the Science and Engineering Research Board, Government of India to Dr. Shagun Aggarwal (SERB File No. EMR/2016/002478).

\section{Author Contributions}

K. Tallapaka performed clinical evaluation and fetal autopsy. S. Aggarwal performed clinical evaluation, ultrasonography, and fetal autopsy. A. Bhattacherjee performed NGS analysis. A. Das Bhowmik performed NGS analysis and Sanger sequencing, and A. Dalal performed NGS analysis. All authors revised and reviewed the work.

\section{Disclosure Statement}

The authors have no conflicts of interest to declare.

\section{References}

Das Bhowmik A, Dalal A: Whole exome sequencing identifies a novel frameshift mutation in GPC3 gene in a patient with overgrowth syndrome. Gene 572:303-306 (2015).

$\checkmark$ Green RC, Berg JS, Grody WW, Kalia SS, Korf BR, et al: ACMG recommendations for reporting of incidental findings in clinical exome and genome sequencing. Genet Med 15:565-574 (2013).

-Kalia SS, Adelman K, Bale SJ, Chung WK, Eng C, et al: Recommendations for reporting of secondary findings in clinical exome and genome sequencing, 2016 update (ACMG SF v2.0): a policy statement of the American College of Medical Genetics and Genomics. Genet Med 19:249-255 (2017).

Khaddour R, Smith U, Baala L, Martinovic J, Clavering $\mathrm{D}$, et al: Spectrum of $M K S 1$ and $M K S 3$ mutations in Meckel syndrome: a genotypephenotype correlation. Hum Mutat 28:523524 (2007).
Li H, Durbin R: Fast and accurate long-read alignment with Burrows-Wheeler transform. Bioinformatics 26:589-595 (2010).

Li H, Handsaker B, Wysoker A, Fennell T, Ruan J, et al: The Sequence Alignment/Map format and SAMtools. Bioinformatics 25:2078-2079 (2009).

Mackley MP, Fletcher B, Parker M, Watkins H, Ormondroyd E: Stakeholder views on secondary findings in whole-genome and wholeexome sequencing: a systematic review of quantitative and qualitative studies. Genet Med 19:283-293 (2017).
McKenna A, Hanna M, Banks E, Sivachenko A, Cibulskis K, et al: The Genome Analysis Toolkit: A MapReduce framework for analyzing next-generation DNA sequencing data. Genome Res 20:1297-1303 (2010).

Richards S, Aziz N, Bale S, Bick D, Das S, et al: Standards and guidelines for the interpretation of sequence variants: a joint consensus recommendation of the American College of Medical Genetics and Genomics and the Association for Molecular Pathology. Genet Med 17:405-424 (2015).

Tabor HK, Auer PL, Jamal SM, Chong JX, Yu JH, et al: Pathogenic variants for Mendelian and complex traits in exomes of 6,517 European and African Americans: implications for the return of incidental results. Am J Hum Genet 95:183-193 (2014)

-Wang K, Li M, Hakonarson H: ANNOVAR: functional annotation of genetic variants from high-throughput sequencing data. $\mathrm{Nu}$ cleic Acids Res 38:e164 (2010). 